en moyenne de 3,9 et $5,2 \mu$. Le calcul du diamètre moyen concordait. avec les données de RAHN.

Le nombre de globules gras dans le beurre est très grand. D'après. les calculs de Boysen [66], il varie pour $1 \mathrm{gr}$. de beurre, entre 9 et. 25 milliards.

(A suivre.)

\title{
CONSIDÉRATIONS GÉNÉRALES SUR LES BABEURRES
}

\section{JEAN PIEN}

Ingénieur chimiste (I. C. R.),

Docteur ès-sciences,

Directeur des Laboratoires

des "Fermiers Réunis". par

et

ROBERT MARTIN

Ingénieur chimiste (I. C. R.),

à la Société des "Fermiers Réunis".

Traviail exécuté en collaboration avec $M$. Bonn, Expert-chimiste près la Cour d'A ppel de Paris et le Tribunal civil de la Seine.

Etant donné l'importance prise par le babeurre en diététique et en thérapeutique infantiles, nous avons eru intéressant de procéder à une étude assez complète de la question.

Nous voulons d'abord dans le présent article rassembler quelques documents généraux sur cette question, concernant, non seulement le babeurre véritable, mais aussi les babeurres médicaux et les babeurres que nous appellerons "reconstitués».

Le but précis de cette première et courte note est d'établir la distinction qui doit exister entre les différentes sortes de babeurres. - sans d'ailleurs pousser très loin l'étude de chacun d'eux.

Nous allons envisager successivement:

$1^{\circ}$ Le babeurre véritable.

$2^{\circ}$ Les babeurres médicaux.

$3^{\circ}$ Les babeurres reconstitués.

$$
*^{*} *
$$

\section{LE BABEURRE VERITABLE.}

I. Définition. - Le babeurre véritable est le produit du barattage de la crème.

VAQUEZ [1] l'appelle "le liquide résiduel du barattage du lait ".

Manquat [2] le désigne comme étant "le lait qui reste après l'extraction du beurre" ".

Le Professeur Poncher [3] le définit comme étant "du lait écrémé modifié par les fermentations lactiques lors de la maturation de la crème ".

Lemoine et GÉraRd [4] considèrent le babeurre comme "le liquide restant après barattage du lait ou de la crème ". 
Demelin et Devraigne [5] le définissent également comme "le petit-lait qui reste après le barattage du lait».

Ch. Renous [6] le désigne comme « le liquide qui reste lorsque le beurre a été extrait complètement du lait de vache où une partie du lactose a été transformée en acide lactique par la fermentation ".

Toutes ces définitions s'accordent à reconnaître au babeurre deux caractères :

$1^{\circ}$ Il est le produit d'un barattage (de lait ou de crème).

$2^{\circ}$ Il résulte d'une fermentation lactique.

Ces deux caractères, d'ailleurs, sont à peu près inséparables ; car, en principe, le barattage d'une crème (ou d'un lait) ne se fait (et ne peut guère se faire en vue de la séparation du beurre) qu'après une fermentation lactique suffisante.

En somme, le babeurre est du petit-lait caillé (par la fermentation lactique) et battu (par le barattage).

Sa source peut être la crème, ou, plus simplement, le lait.

II. Préparation. - En règle générale, donc, le babeurre s'obtient par le barattage de la crème acidifiée soit spontanément, soit par addition de ferments lactiques (après pasteurisation).

Les facteurs qui influencent la production du babeurre (quantité, composition) sont ceux-là même qui influencent le barattage. Il y a done lieu d'en dire quelques mots.

La crème extraite normalement du lait par écrémage centrifuge est un mélange de matière grasse pure et de petit-lait. Le barattage, qui a pour but de rassembler la matière grasse pure, libère donc, en principe, le lait écrémé contenu dans la crème. En fait, le babeurre a bien la même composition globale que le lait écrémé (teneurs en caséine, lactose, cendres). Mais il y a lieu de tenir compte de l'influence sur la composition du babeurre de ces "facteurs" du barattage.

1. Influence de 1'eau ajoutée. - On sait que lors du barattage on ajoute à la crème (à un moment qu'il importe de bien saisir) une certaine quantité d'eau. Bien entendu cette eau se retrouve dans le babeurre qui, de ce fait, est toujours plus dilué que le petit-lait correspondant. Cette addition d'eau, en cours de barattage, augmente la quantité de babeurre produite, mais diminue l'extrait sec du babeurre. En effet :

a) Influence sur la quantité de babeurre obtenu:

L'excès de la quantité de babeurre produite n'est pas, comme on pourrait le supposer, égal à la quantité d'eau introduite, mais souvent supérieur.

Ainsi, dans des conditions variées, nous avons obtenu (volume du babeurre \% du volume de crème) : 


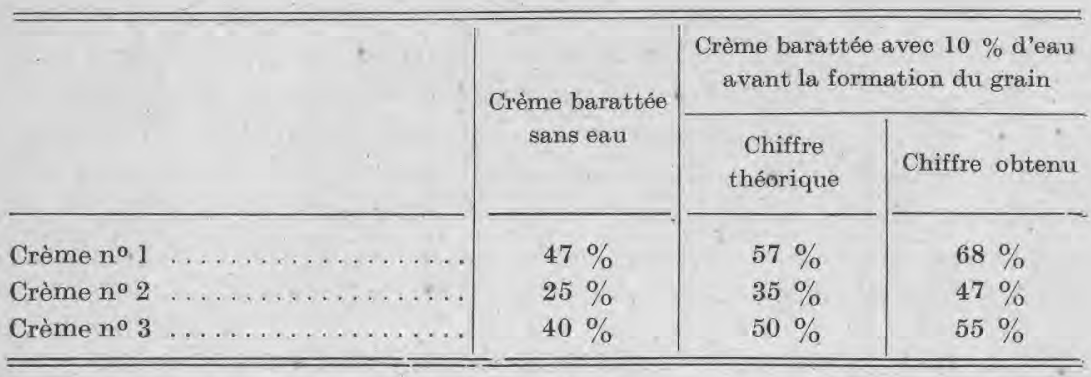

Done : l'addition d'eau en cours de barattage favorise grandement. l'extraction du babeurre et en libère un volume supérieur au volume théorique (sans eau) majoré de la quantité d'eau introduite.

b) Influence sur l'extrait sec du babeurre:

En reprenant les exemples précédents on obtient (extraits en grammes par litre de babeurre) :

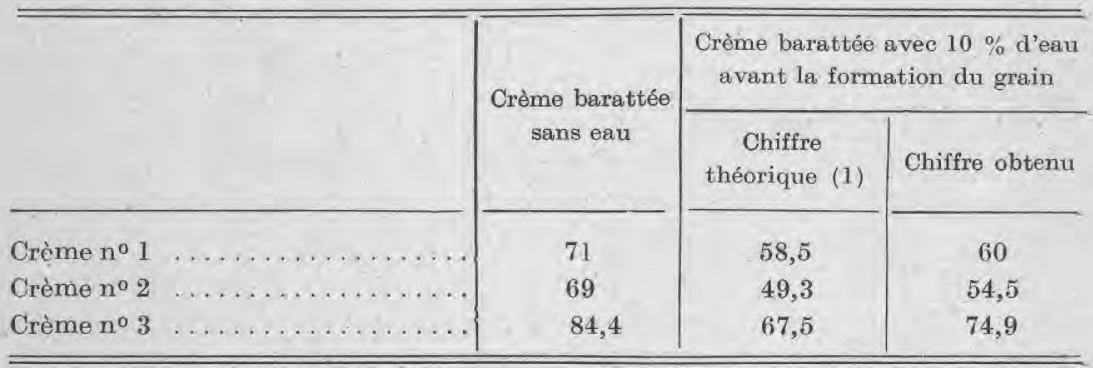

(1) Calculé compte tenu des volumes réels de crème mise en ceuvre et de babeurre obtenu sans addition d'eau.

Done : l'addition d'eau fournit un babeurre plus riche (compte. tenu de la dilution) qu'un barattage sans addition d'eau.

C'est en somme une autre manifestation de ce phénomèné de la meilleure extraction du babeurre par barattage en présence d'eau.

$2^{\circ}$ Influence de l'acidité de la crème mise en œuvre. - L'acidité de la crème a, on le sait, une grande influence sur la façon dont elle se comporte au barattage. Le barattage est d'autant plus rapide que la crème est plus acide. Mais aussi, l'extrait sec du babeurre obtenu est d'autant plus élevé que la crème soumise au barattage est plus acide.

Ainsi, des barattages effectués sur une même crème ont donné :

\begin{tabular}{|c|c|c|c|}
\hline $\begin{array}{c}\text { Acidité de la crème au moment du } \\
\text { barattage } \ldots \ldots \ldots \ldots \ldots \ldots \ldots \ldots\end{array}$ & $25^{\circ}$ Dornic & $37^{\circ}$ Dornic & $48^{\circ}$ Dornic \\
\hline Durée du barattage ............. & 51 minutes & 40 minutes & 26 minutes \\
\hline Extrait sec du babeurre $\ldots . \ldots \ldots \ldots$ & 56,5 & 58,3 & 70,3 \\
\hline
\end{tabular}


$3^{\circ}$ Remarques. - En ce qui concerne la teneur du babeurre en matière grasse résiduelle, nous avons observé que l'addition d'eau (en dehors de l'influence de la dilution) avait une action favorable dans le sens de la production d'un babeurre moins gras. Il semble aussi qu'un babeurre est d'autant moins gras que la crème mise en œuvre est plus acide. Mais on n'a pas encore découvert les lois qui régissent l'enrichissement ou l'appauvrissement des babeurres en matière grasse au cours du barattage.

III. Composition. - La composition du babeurre normal (issu du barattage de la crème) est exactement, à la dilution près, celle du lait écrémé provenant du lait entier mis en œuvre. Les teneurs en easéine, lactose, matières minérales sont celles du petitlait multipliées par le rapport des extraits du babeurre et du petitlait. Il est à remarquer que dans bien des cas, même en barattant sans aucune addition d'eau, le babeurre obtenu a un extrait total inférieur à celui du petit-lait issu du même lait. Cela tient, comme nous l'avons dit ci-dessus, à d'autres circonstances (par exemple l'acidité), qui font que l'extraction est plus ou moins parfaite. De toute façon, l'extrait sec normal d'un babeurre véritable ne peut guère atteindre (et encore moins dépasser) 80 à 85 gr. par litre dans les cas les plus avantageux.

La densité des babeurres est évidemment fonetion de l'extrait total et croît à peu près comme lui. A titre documentaire, voici quelques comparaisons entre l'extrait et la densité de babeurres véritables :

\begin{tabular}{c|c|c|c|c|c|c}
\hline \hline & \multicolumn{5}{|c}{ Désignation des crèmes } \\
\cline { 2 - 6 } & $\mathrm{A}$ & $\mathrm{B}$ & $\mathrm{C}$ & $\mathrm{D}$ & $\mathrm{E}$ & $\mathrm{F}$ \\
\hline & & & & & & \\
$\begin{array}{c}\text { Densité du babeurre } \\
\text { (avee } 10 \% \text { d'eau) } \ldots\end{array}$ & 1.013 & $1.017,5$ & 1.018 & 1.022 & 1025 & $1.027,5$ \\
$\begin{array}{c}\text { Extrait total de ces } \\
\text { babeurres ........ }\end{array}$ & 40 & 54 & 53,6 & 65,8 & 65,3 & 85 \\
\hline \hline
\end{tabular}

L'acidité d'un babeurre est toujours supérieure à celle de la crème qui lui a donné naissance. Il est facile de le concevoir ; l'acidité d'une crème étant exclusivement contenue dans la partie non grasse de la crème, au barattage, la matière grasse à peu près pure se trouve isolée et toute l'acidité de la crème passe dans un volume de babeurre plus faible.

Nous avons obtenu par exemple (en degrés Dornic): 


\begin{tabular}{|c|c|c|c|c|}
\hline & \multicolumn{4}{|c|}{ Désignation des crèmes } \\
\hline & A & B & C & D \\
\hline Acidité des crèmes $\ldots \ldots \ldots \ldots \ldots \ldots \ldots$ & 31,5 & 35 & 40 & 52 \\
\hline Acidité du babeurre produit sans eau ..... & 50 & 50 & 55 & 64 \\
\hline $\begin{array}{r}\text { Acidité du babeurre produit avec } 10 \% \\
\text { d'eau } \ldots \ldots \ldots \ldots \ldots \ldots \ldots \ldots \ldots \ldots\end{array}$ & 45 & 43 & 47 & 55 \\
\hline
\end{tabular}

La matière grasse des babeurres, avons-nous dit, varie beaucoup. Il est important, dans une beurrerie, d'en faire la détermination exacte et fréquente, car il peut y avoir là une cause importante de pertes de matière grasse. Suivant les conditions de la fabrication (acidité de la crème; température, eau introduite) la richesse des babeurres en matière grasse peut varier de 0 à 5,6 et même $10 \mathrm{gr}$. par litre. Il est d'ailleurs possible d'obtenir industriellement du babeurre véritable pratiquement exempt de matière grasse.

Remarques. - D'après MARRE [7], le babeurre contiendrait une proportion de lécithine très supérieure à celle du lait entier initial. Ce fait ne pourrait s'expliquer que par une accumulation des lécithines dans la crème au cours de l'écrémage et par un abandon de ces substances dans le babeurre au cours du barattage.

IV. Caractères du babeurre normal. - Le babeurre, avonsnous dit, résulte du barattage d'une crème acidifiée, c'est-à-dire ayant subi la fermentation lactique.

Les deux caractères principaux du babeurre normal sont done les suivants :

$1^{\circ}$ Présence d'acide lactique et de ferments lactiques. L'acidité lactique d'un babeurre est éminemment variable suivant qu'il provient d'une crème plus ou moins acide. Dans la pratique industrielle, il y a intérêt à fixer les conditions de la maturation de telle manière que cette acidité soit constante.

Si on a opéré sur une crème acidifiée spontanément on risque d'avoir, à côté des ferments lactiques ordinaires de la crème, de nombreux autres germes dont certains peuvent être indésirables non seulement pour les qualités organoleptiques du babeurre, mais aussi et surtout pour ses qualités hygiéniques.

En opérant au contraire sur de la crème pasteurisée, réensemencée d'une culture pure de ferments lactiques, on peut obtenir (sous réserve de réaliser un barattage aseptique) des babeurres ne contenant que des ferments lactiques.

$2^{\circ}$ Etat physico-chimique particulier de la caséine. - Il 
résulte des travaux de FrevdenReich et de ceux de Mazé que les ferments lactiques solubilisent partiellement la caséine même en milieu acide. Dans la maturation de la crème, cette action se produit parallèlement à l'action coagulante et, dans le babeurre normal, on recueille une suspension de caséine qui possède les trois caractères suivants :

a) Elle est coagulée (par l'acidification).

b) Elle est partiellement solubilisée (par les ferments lactiques).

c) Elle est finement divisée (par le barattage).

Le caractère principal du babeurre est vraisemblablement cet état physico-chimique particulier de la caséine fine, divisée et partiellement « digérée ». Un babeurre de préparation récente (possédant par exemple une acidité de $70^{\circ}$ D.) présente l'aspect d'une " huile " blanche sans grumeaux, dont les grains de caséine sont à peine visibles à l'œil nu et ne se déposent d'ailleurs que lentement.

La chaleur ne rassemble que d'une façon imparfaite cette suspension de caséine et ne produit pas le précipité grossier qu'on observe en chauffant un lait sur le point de cailler.

Même, l'addition d'un acide quelconque ne provoque aucune précipitation au sein d'un babeurre véritable. La caséine est, en effet, déjà précipitée, mais sous une forme particulière qui la rend peu visible et difficile à rassembler en masses volumineuses.

Bien entendu l'obtention de babeurres possédant ces caractères nécessite l'usage des crèmes ayant subi une fermentation lactique véritable et prolongée ( 24 heures au moins).

$3^{\circ}$ Remarque. - Il est logique de penser que la plupart des vitamines hydrosolubles du lait (et certaines des vitamines liposolubles) se retrouvent dans le babeurre, à condition que le lait et la crème n'aient pas été portés à une température susceptible de les détruire. La preuve directe en a d'ailleurs été faite par des expériences biologiques sur des animaux.$$
*^{*} *
$$

\section{LES BABEURRES MÉDICAUX.}

Les caractères précédents (et surtout l'état de "prédigestion " de la caséine) ont depuis longtemps fait du babeurre un produit thérapeutique des plus intéressants pour l'enfance et pour certains malades.

L'intolérance de beaucoup d'enfants vis-à-vis du lait provient, bien souvent, de ce fait que le caillage du lait dans l'estomac provoque la formation d'une masse volumineuse et compacte de caséine, peu perméable aux sucs digestifs. L'usage de ferments végétaux permettant de réaliser dans les biberons une coagulation douce 
instantanée que l'on fait suivre d'une agitation violente pour briser la caséine, apportent déjà une sérieuse amélioration dans l'alimentation de certains enfants supportant mal le lait. Le babeurre, avec sa caséine déjà coagulée, finement divisée et même partiellement solubilisée, est, selon la majorité des pédiâtres, un aliment de premier ordre dans les eas d'intolérance vis-à-vis du lait.

I. Babeurres médicaux stérilisés-farinés. - Mais la production d'un babeurre frais, immédiatement consommable, exempt de germes pathogènes, a posé un problèmé industriel évidemment difficile à résoudre. On a tenté de tourner la difficulté en stérilisant le babeurre et certains industriels fabriquent journellement des babeurres stérilisés.

Ici, une remarque très importante s'impose : La stérilisation pure et simple du babeurre normal est une opération délicate. Nous disions ci-dessus que, si la chaleur n'a pas pour résultat de réaliser une précipitation massive et grossière de la caséine du babeurre, du moins a-t-elle tendance à "rassembler" la caséine du babeurre (d'une façon imparfaite d'ailleurs). La stérilisation exagère ce phénomène. En fait, le maintien prolongé à une haute température rend peu à peu la caséine grenue et, bien qu'on n'atteigne jamais complètement l'aspect de "lait caillé ", on risque certainement de perdre une partie des précieuses qualités de ce produit.

On a donc cherché à "stabiliser » les babeurres pour leur permettre de subir la stérilisation: On a essayé d'y parvenir en additionnant le babeurre d'amidon. Le chauffage détermine la formation d'un empois, qui paraît empêcher la caséine de se rassembler et d'autant mieux qu'il y a plus d'amidon. Le mécanisme de cette action, que nous nous sommes, pour le moment, contentés d'observer comme les industriels qui l'utilisent, peut-il s'expliquer par la théorie des solutions colloïdales? En tout cas, la réussite de cette "stabilisation" exige un tour de main particulier, sans lequel on n'obtient pas le résultat attendu.

Certains industriels ont done lancé le babeurre liquide stériliséfariné (et sucré), que l'on trouve couramment dans le commerce. Cette addition d'hydrates de carbone a été estimée utile par certains pédiâtres et combattue par d'autres. En fait, à l'heure actuelle, il n'est guère utilisé comme babeurres médicaux autre chose que des babeurres stérilisés additionnés ou non de farines, de sucre et quelquefois de beurre.

II. Composition des babeurres médicaux. - Voici, à titre documentaire, la composition détaillée de deux babeurres du commerce analysés par nous : 


\begin{tabular}{|c|c|c|}
\hline & \multicolumn{2}{|c|}{ Composition ramenée au litre } \\
\hline & A & B \\
\hline 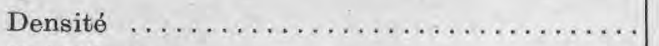 & 1.092 & $1.045,4$ \\
\hline Extrait sec $\ldots \ldots \ldots \ldots \ldots \ldots \ldots \ldots \ldots \ldots \ldots \ldots \ldots$ & 178 & 138 \\
\hline Caséine $\ldots \ldots \ldots \ldots \ldots \ldots \ldots \ldots \ldots \ldots \ldots \ldots \ldots \ldots \ldots \ldots$ & 31,4 & 22,7 \\
\hline Lactose ...................... & 43,4 & 39,5 \\
\hline Matière grasse ......................... & 1,5 & 3,5 \\
\hline Acidité libre (en acide lactique) $\ldots \ldots \ldots \ldots$ & 3,7 & 4,4 \\
\hline Matières minérales.$\ldots \ldots \ldots \ldots \ldots \ldots \ldots \ldots$ & 8,0 & 7,6 \\
\hline Saccharose $\ldots \ldots \ldots \ldots \ldots \ldots \ldots \ldots \ldots$ & 56,3 & 31,6 \\
\hline Amidons $\ldots \ldots \ldots \ldots \ldots \ldots \ldots \ldots \ldots \ldots \ldots$ & 33,7 & 28,7 \\
\hline $\begin{array}{l}\text { Ce qui correspond à peu près à la composition } \\
\text { globale suivante }: \ldots \ldots \ldots \ldots \ldots \ldots \ldots \ldots\end{array}$ & & \\
\hline Extrait du babeurre initial. ................ & $88,0(1)$ & 77,7 \\
\hline Saccharose $\ldots . . \ldots \ldots \ldots \ldots \ldots \ldots \ldots \ldots \ldots$ & 56,3 & 31,6 \\
\hline Amidons $\ldots \ldots \ldots \ldots \ldots \ldots \ldots \ldots \ldots \ldots$ & 33,7 & 28,7 \\
\hline
\end{tabular}

(1) Ce chiffre élevé semblerait indiquer que cette préparation n'est pas à base de babeurre véritable de crème, mais à base de babeurre de lait (voir ci-après).

Nous pourrions eiter de nombreux exemples d'analyses ou de formules de babeurres médicaux. La littérature médicale est riche de ees formules, qui ne diffèrent entre elles que par les proportions respectives des constituants.

III. Remarques. - Si la pratique courante semble avoir complètement rejeté le babeurre frais pour ne prescrire que des babeurres stérilisés (et farinés), il est très vraisemblable de penser que c'est par suite de l'impossibilité où se trouvait le corps médical de se procurer des babeurres frais exempts de germes pathogènes. Quand le docteur RIBADEAU-DUmas [8], vantant les mérites du babeurre, écrit : "... le babeurre est, après le lait de femme, la substance qui contient les protéines les plus favorables pour la nutrition des jeunes enfants ", nous pensons que cette appréciation, si elle s'applique aux babeurres stérilisés, doit, à fortiori, s'appliquer à des babeurres frais présentant toutes les garanties nécessaires tant au point de vue de l'hygiène qu'au point de vue de la conservabilité.

Nous pourrions même aller plus loin et dire que, à certains points de vue, les babeurres frais (provenant de crèmes pasteurisées et ensemencées) et exempts de germes autres que les ferments lactiques, doivent présenter, à priori, certains avantages sur les babeurres stériles (intérêt des vitamines au point de vue nutrition, intérêt des ferments lactiques vivants au point de vue thérapeutique des affec- 
tions gastro-intestinales). Nous nous proposons de poursuivre cette étude (et notamment celle de la production industrielle de babeurre frais et sain) et de préciser certains des avantages qui pourraient résulter de la substitution de celui-ci aux babeurres stérilisés du commerce.

\section{** * \\ III. LES BABEURRES EN POUDRE ET LES BABEURRES RECONSTITUÉS.}

Le séchage du babeurre frais (1) est une opération relativement facile sur cylindres, sous réserve de fixer judicieusement la température de la vapeur et le débit du babeurre sur les rouleaux. Mais les quantités produites sont relativement minimes.

En effet : 1.000 litres de lait entier (à 40 gr. de matière grasse) donnent théoriquement 100 litres de crème à $400 \mathrm{gr}$. de matière grasse par litre. Cette crème contient, en puissance, environ 60 litres de babeurre frais (théoriquement) et 5 à $6 \mathrm{~kg}$. de babeurre en poudre. En pratique les chiffres obtenus sont nettement plus faibles.

L'opération est, comme on le voit, assez onéreuse et certains industriels étrangers ont cherché à tourner la difficulté en fabriquant d'abord du lait écrémé en poudre par les moyens ordinaires (lait frais non acide) et en incorporant à cette poudre sous une forme convenable de l'acide lactique pur (mélangé à de la caséine ou vaporisé sur la poudre de lait). On réalise ainsi un mélange ayant l'acidité du babeurre en poudre véritable et une composition chimique globale très voisine de celle du babeurre vrai. Bien entendu la caséine contenue dans un tel produit n'a jamais subi la fermentation lactique et n'a pas les caractères physico-chimiques de celle du babeurre de barattage de crème mûrie. Ces produits ne sont done pas comparables aux babeurres véritables, dont ils n'ont pas les qualités. Hâtons-nous de dire d'ailleurs qu'ils ne sont pas vendus comme babeurres médicaux, mais comme babeurres pour l'élevage (aviculture surtout). Il y a cependant intérêt à pouvoir les reconnaître et à les distinguer des autres. Nous indiquerons ultérieurement le moyen qui nous a paru le plus convenable.

En tout cas il ne saurait être question de retenir ces produits dans une étude d'ensemble sur les babeurres, puisqu'ils n'ont de ces derniers que la composition globale, mais non pas la véritable strueture.

\section{BIBLIOGRAPHIE.}

[1] Vaquez. Précis de thérapeutique, Paris, 1907.

[2] Manquat. Traité élémentaire de thérapeutique, Paris, 19]2, 2, 335.

(1) Par frais nous entendons bien babeurre acide, mais non stérile, et de préparation récente. 
[3] Ch. Poncher. Conférence du 27 janvier 1918 à la Société d'Hygiène alimentaire.

[4] Lemoine et Gérard. Formulaire de consultations médicales et chirurgicales, Paris, 1909.

[5] Demelin et Devratgne. Manuel du puériculteur, Paris, 1921.

[6] Ch. Renous. Thèse Doct. méd., Paris, 1924. Legrand, éditeur.

[7] F. Marre. Revue générale de chimie pure et appliquée, février 1911, 14, 56.

[8] Dr Ribadeau-Dumas, Mathieu et Guìde. Bulletin de la Société de Pédiatrie de Paris, décembre 1931, 569.

\title{
ÉTUDE COMPARATIVE DES PRINCIPALES CONSTANTES UTILISÉES POUR LA DÉTERMINATION DU MOUILLAGE DU LAIT
}

\author{
par J. CERF
}

Ingénieur de l'Institut agricole et diplômé de l'Ecole de Laiterie de la Faculté des Sciences de Nancy, Ancien assistant de l'Etablissement fédéral d'Industrie laitière et de Bactériologie du Liebefeld, Berne.

(Fin)

\section{LA CONSTANTE BOUIN \\ OU $\mathbf{L}+5 \mathbf{C}=$ LACTOSE + 5 FOIS LES CENDRES.}

Origine. - Nous avons vu, au début de la $2^{\mathrm{e}}$ partie de notre étude, quelles sont les variations des composants du lait au cours de la lactation. Un coup d'œil sur le graphique nous montre que les cendres brutes et le lactose ont les variations les plus faibles, soit de 18 à $20 \%$. Ces variations ne se manifestent pas dans le même sens. Alors que le lactose diminue, les cendres brutes augmentent, et cela dans la même proportion. Mais les cendres ne représentent que 1/7 du lactose. C'est en se basant sur ces observations que M. Bourn proposa sa constante. Tout naturellement nous nous demandons pourquoi M. BouIn a choisi le facteur 5 pour multiplier les cendres, et non le facteur 7. L'expérience a démontré que le facteur 7 était un peu trop fort, par suite des variations des cendres, qui sont proportionnellement plus fortes que celles du lactose. Le graphique (période colostrale à part) nous indique une variation de 17 à $18 \%$ du lactose et 21 à $22 \%$ des cendres. En calculant, nous trouvons que cette constante joue à merveille. Ainsi, les cendres oscillant de 6,7 à 8,5 , nous comptons une augmentation de 1 gr. 8. D'autre part, le lactose diminue de 9 gr.

Donc, 1,8 gr. $\times 5=9,0$ gr. augmentation de la constante Diminution du lactose $=9,0 \mathrm{gr}$.

Différence $\overline{=0,0 \mathrm{gr} \text {. }}$ 